\title{
Perception of Polynomial for Weighted Directed Graph
}

\author{
Hend El- Morsy \\ Correspondence: Hend El-Morsy, College of the Preparatory Year, Department of Mathematics, Umm Al-Qura \\ University, Mecca, Kingdom of Saudi Arabia. E-mail: heebrahim@uqu.edu.sa, hendelmorsy@yahoo.com
}

Received: October 5, 2019 Accepted: November 18, 2019 Online Published: November 20, 2019

doi:10.5539/jmr.v11n6p84

URL: https://doi.org/10.5539/jmr.v11n6p84

\begin{abstract}
In this paper we will apply a polynomial for directed weighted graph. We will introduce notion of deletion and contraction in directed weighted graph. Some examples and propositions will be illustrated.
\end{abstract}

Keywords: directed graph, weighted graph, contraction, deletion, polynomials

\section{Introduction}

In mathematics, a polynomial is an expression consisting of variables (also called indeterminates) and coefficients, that involves only the operations of addition, subtraction, multiplication, and non-negative integer exponents of variables. An example of a polynomial of a single indeterminate, $x$, is $x^{2}-4 x+7$. An example in three variables is $x^{3}+2 x y z^{2}-y z+1$.

Polynomials appear in many areas of mathematics and science. For example, they are used to form polynomial equations, which encode a wide range of problems.

Let $\vec{G}_{\mathrm{w}}(\mathrm{V}, \mathrm{A})$ be a directed weighted graph, a directed weighted polynomial of $\vec{G}$ maping $\mathrm{C}$ from $\mathrm{V}$ to the set of $\mathrm{X}_{\mathrm{n}}$ satisfying :

$$
\text { i. } \forall(\mathrm{x}, \mathrm{y}) \boldsymbol{\epsilon} \mathrm{A}, \overrightarrow{\mathrm{xy}} \neq \mathrm{yx} \text {. }
$$

ii. A weighted graph consists of finite graph $\vec{G}$ with vertex set $\left\{\mathrm{v}_{1}, \mathrm{v}_{2}, \ldots \ldots \mathrm{v}_{\mathrm{n}}\right\}$, edge set $\mathrm{E}$ together with weight function $\mathrm{W}: \mathrm{V} \rightarrow \mathrm{Z}^{+}$then $\mathrm{W}\left(\mathrm{V}_{\mathrm{i}}\right)$ the weight of $\mathrm{V}_{\mathrm{i}}$.

iii. If $\mathrm{U} \subset \mathrm{V}$ we define weight of $\mathrm{U}, \mathrm{W}(\mathrm{U})$ to be $\sum_{v \in V} W(v)$

\section{Polynomial for Weighted Directed Graph}

We need to introduce notion of deletion and contraction in directed weighted graph $\vec{G}_{\text {w }}$ as follows:

* If e is edge of $(\overrightarrow{\mathrm{G}}, \mathrm{w})$, then let $\left(\vec{G}_{\mathrm{e}}\right.$, w) denote the graph obtained from $\overrightarrow{\mathrm{G}}$ by deleting e and leaving weight unchanged, see Fig.(1)

*If e is an edge of simple directed weighted graph $(\vec{G}, w)$, then $\left(\vec{G}_{e}^{c}, w\right)$ is graph formed from $\left(\vec{G}_{e}^{\Uparrow}\right.$, w) by replacing every parallel class by single edge. Fig. (1)

*If e is not loop of $(\mathrm{G}, \mathrm{w})$, then let $\left(\vec{G}^{\|}\right.$, w) be a graph obtained by contracting e that is deleting identifying its end points $\mathrm{V}, \mathrm{V}^{\prime}$ into a single vertex $\mathrm{V}^{\prime \prime}$ and setting $\mathrm{W}\left(\mathrm{V}^{\prime \prime}\right)=\mathrm{W}(\mathrm{V})+\mathrm{W}\left(\mathrm{V}^{\prime}\right)$ if the edges in the same direction Fig. $(2-\mathrm{a})$, and $\mathrm{W}\left(\mathrm{V}^{\prime \prime}\right)=$ $\mathrm{W}(\mathrm{V})-\mathrm{W}\left(\mathrm{V}^{\prime}\right)$ if the edges in opposite directions. Fig. $(2, \mathrm{~b})$.
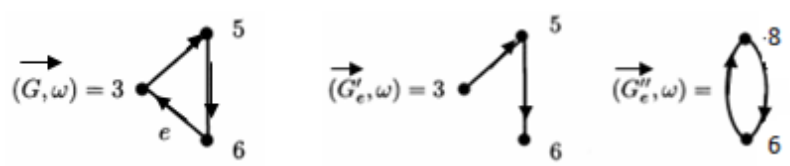

$\overrightarrow{\left(G_{e}^{c}, \omega\right)}=\mathfrak{f}_{6}^{8}$

Figure 1. 

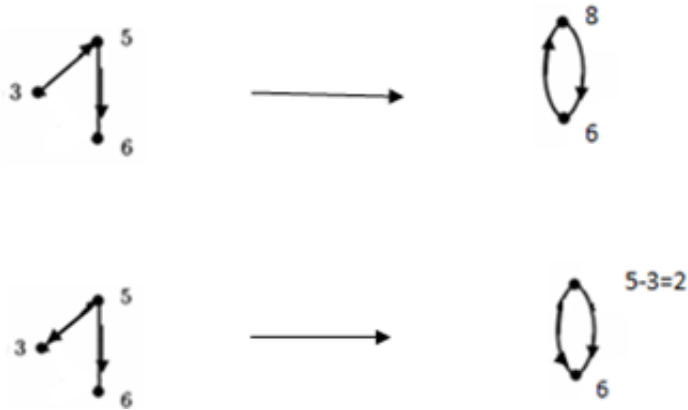

Figure 2-a.

We associate with any directed weighted graph $(\vec{G}, \mathrm{~W})$, a multivariate polynomial $\mathrm{W}_{\mathrm{G}}(\mathrm{x}, \mathrm{y})$ which define as follows: Let $\mathrm{y}_{1}, \mathrm{x}_{1} \mathrm{x}_{2}, \ldots \ldots \mathrm{x}_{\mathrm{n}}$ be commuting indeterminates.

Now let $\mathrm{W}_{\mathrm{G}}(\mathrm{x}, \mathrm{y})$ be defined recursively by the following rules:

i. If $\vec{G}_{\mathrm{W}}$ consists of $\mathrm{m}$ isolated vertices with weights $\mathrm{w}_{1}, \mathrm{w}_{2} \ldots \ldots \mathrm{w}_{\mathrm{m}}$ then $\mathrm{W}_{\mathrm{G}}(\mathrm{x}, \mathrm{y})=\mathrm{X}_{\mathrm{w} 1} \ldots \ldots \mathrm{X}_{\mathrm{Wm}}$.

ii. If $\vec{G}_{\mathrm{w}}$ has loop, then $\mathrm{W}_{\mathrm{G}}(\mathrm{x}, \mathrm{y})=\mathrm{y} \mathrm{W}_{\mathrm{Gle}}(\mathrm{x}, \mathrm{y})$.

iii. The polynomial take the form: $X_{n} X_{m}+X_{z}+X_{z} y,(z=n+m)$.

\section{Example2.1:}

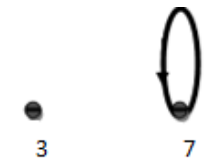

If $(\vec{G}, \mathrm{~W})=$

Then $\mathrm{W}_{\mathrm{G}}(\mathrm{x}, \mathrm{y})=\mathrm{X}_{3} \mathrm{X}_{7} \mathrm{y}$ ( $(\mathrm{G}$ a loop $)$

b. If $(\vec{G}, \mathrm{~W})=$
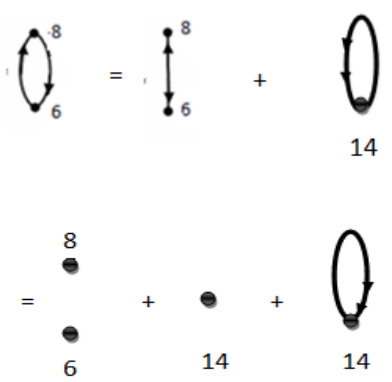

$=\mathrm{X}_{8} \mathrm{X}_{6}+\mathrm{X}_{14}+\mathrm{X}_{14} \mathrm{Y}$.

Theorem 2.2:

Let $\vec{G}(\mathrm{~V}, \mathrm{~W})$ be weighted directed graph, and let $\overrightarrow{\mathrm{G}}_{1}, \overrightarrow{\mathrm{G}}_{2}$ be two non-empty subsets of G, such that $\vec{G}=\overrightarrow{\mathrm{G}}_{1} \mathrm{U} \overrightarrow{\mathrm{G}}_{2}$, and if

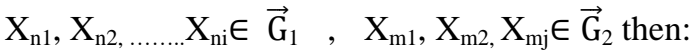

$\mathrm{X}_{\mathrm{wn}} \mathrm{X}_{\mathrm{wm}}=\sum_{i=w}^{n} \sum_{j=w}^{m} x_{\mathrm{i}} \mathrm{X}_{\mathrm{j} \epsilon \mathrm{G}}$

Then we have :

$\mathrm{P}(\vec{G}, \mathrm{~W})=\mathrm{P}\left(\vec{G}_{1}, \mathrm{~W}_{1}\right) \odot \mathrm{P}\left(\vec{G}_{2}, \mathrm{~W}_{2}\right) . \quad$ (Where $\mathrm{P}$ is the related polynomial).

Proof:

Let $\overrightarrow{\mathrm{G}}$ (V, A ) be a weighted directed graph, $\overrightarrow{\mathrm{G}}_{1}, \overrightarrow{\mathrm{G}}_{2}$ are subsets of $\mathrm{G}$ such that $\overrightarrow{\mathrm{G}}=\vec{G}_{1} \mathrm{U} \vec{G}_{2}$

If $\vec{G}_{1}=X_{n 1} X_{n 2} \ldots . . X_{n i}+X_{n 11} X_{n 21}+X_{n \| 1}+X_{n \| 1} Y_{i}$

$\vec{G}_{2}=X_{m 1} X_{m 2} \ldots . . X_{m j}+X_{m 11} X_{m 21}+X_{m \| 1}+X_{m \| 1} Y_{j}$ 


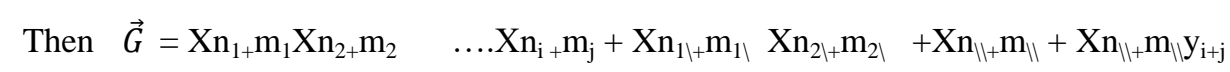

It follows that this polynomial can be found in its factorial form by taken the factorial forms of $\mathrm{X}_{\mathrm{n}}$ and $\mathrm{X}_{\mathrm{m}}$ and adding there as if the factorials were weights.

This process that we denoted symbolically by $\vec{G}_{1} \odot \vec{G}_{2}$.

\section{Example 2.3:}

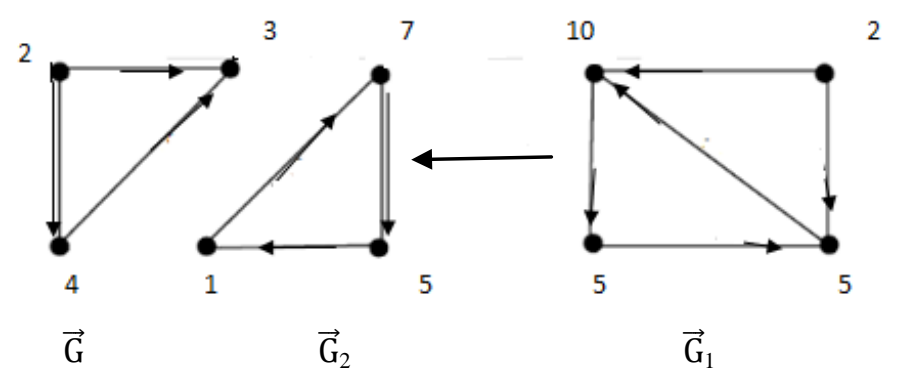

Weighted directed graph $\overrightarrow{\mathrm{G}}, \overrightarrow{\mathrm{G}}_{1}, \overrightarrow{\mathrm{G}}_{2}$ $\ni \overrightarrow{\mathrm{G}}=\overrightarrow{\mathrm{G}}_{1} \mathrm{U} \overrightarrow{\mathrm{G}}_{2}$

First we find polynomial of $\vec{G}_{1}$ and $\vec{G}_{2}$

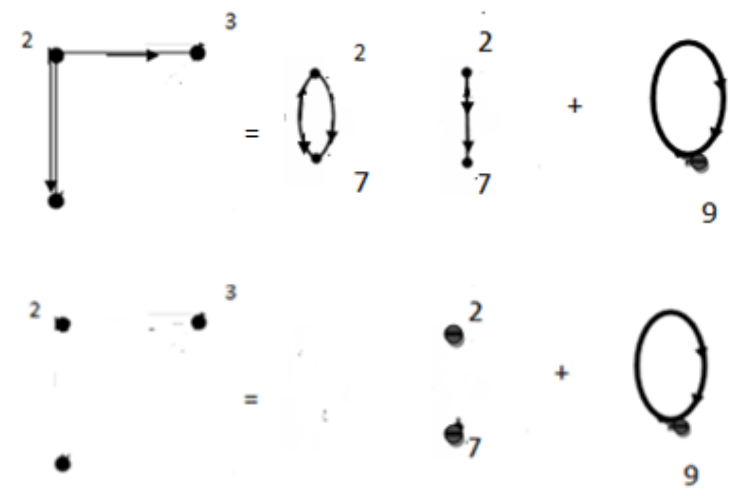

$\mathrm{P}\left(\vec{G}_{1}\right)=\mathrm{X}_{4} \mathrm{X}_{2} \mathrm{X}_{3}+\mathrm{X}_{2} \mathrm{X}_{7}+\mathrm{X}_{9}+\mathrm{X}_{9} \mathrm{Y}$.

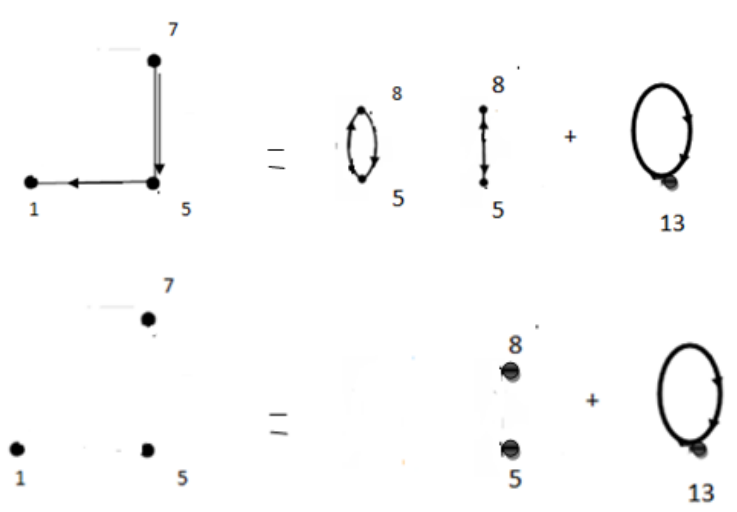

$\mathrm{P}\left(\overrightarrow{\mathrm{G}}_{2}\right)=\mathrm{X}_{7} \mathrm{X}_{5} \mathrm{X}_{1}+\mathrm{X}_{5} \mathrm{X}_{8}+\mathrm{X}_{13}+\mathrm{X}_{13} \mathrm{Y}$

Then $\mathrm{P}(\overrightarrow{\mathrm{G}})=\mathrm{P}\left(\overrightarrow{\mathrm{G}}_{1}\right)+\mathrm{P}\left(\overrightarrow{\mathrm{G}}_{2}\right)$

$\mathrm{P}(\overrightarrow{\mathrm{G}})=\mathrm{X}_{4} \mathrm{X}_{2} \mathrm{X}_{3}+\mathrm{X}_{2} \mathrm{X}_{7}+\mathrm{X}_{9}+\mathrm{X}_{9} \mathrm{Y}+\mathrm{X}_{7} \mathrm{X}_{5} \mathrm{X}_{1}+\mathrm{X}_{5} \mathrm{X}_{8}+\mathrm{X}_{13}+\mathrm{X}_{13} \mathrm{Y}$

$=\mathrm{X}_{5} \mathrm{X}_{7} \mathrm{X}_{10}+\mathrm{X}_{7} \mathrm{X}_{15}+\mathrm{X}_{22}+\mathrm{X}_{22} \mathrm{Y}_{2}$. 


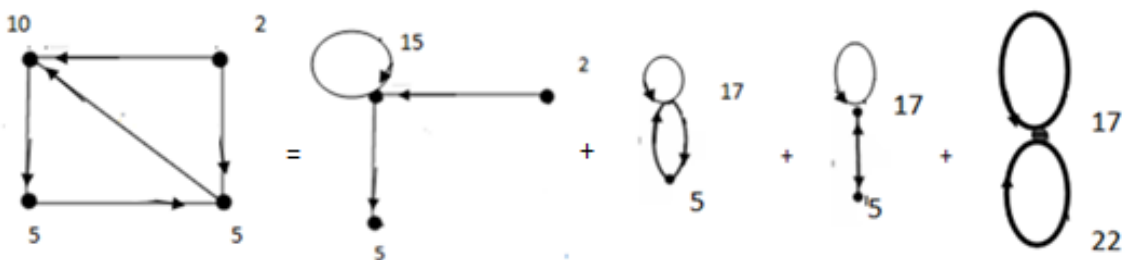

$\mathrm{P}(\vec{G})=\mathrm{X}_{2} \mathrm{X}_{15} \mathrm{X}_{5}+\mathrm{X}_{17} \mathrm{X}_{5}+\mathrm{X}_{22}+\mathrm{X}_{22} \mathrm{Y}_{2}$.

The weights of $X$ in all terms are equal.

Proposition 2.4:

For any weighted directed graph $\vec{G}(\mathrm{~V}, \mathrm{~W})$ with n vertices, we have:

The coefficient of $X_{n} X_{m} \ldots .+X_{n} Y_{m m}$ are 1 .

ii. Polynomial $\mathrm{P}$ ( $\vec{G}, \mathrm{~W}$ ) has no constant term.

iii. Loop write only on the last term with the final $\mathrm{X}_{\mathrm{n}}$ remaining.

Example 2.5:

For weighted directed cycle graph $\mathrm{C}_{6}$ We can compute polynomial as follows:

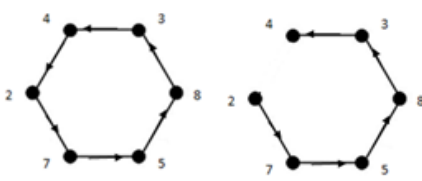

1. $X_{2} X_{9}+X_{11}+X_{11} Y$

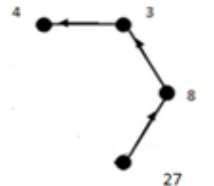

3. $\mathrm{X}_{27} \mathrm{X}_{35}+\mathrm{X}_{62}+\mathrm{X}_{62} \mathrm{Y}$

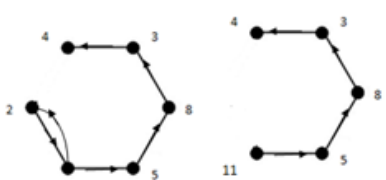

2. $\mathrm{X}_{11} \mathrm{X}_{16}+\mathrm{X}_{27}+\mathrm{X}_{27} \mathrm{Y}$

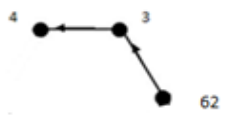

4. $\mathrm{X}_{62} \mathrm{X}_{3}+\mathrm{X}_{65}+\mathrm{X}_{65} \mathrm{Y}$
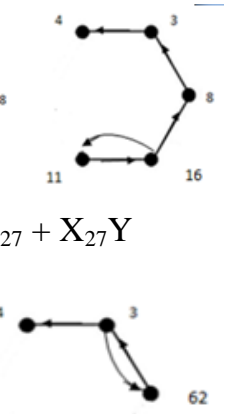

65

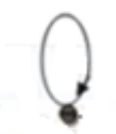

$$
\text { 5. } \mathrm{X}_{4} \mathrm{X}_{65}+\mathrm{X}_{69}+\mathrm{X}_{69} \mathrm{Y}
$$

By adding the five equations we obtain the polynomial of $\mathrm{C}_{6}$ as follows:

$\mathrm{X}_{106} \mathrm{X}_{128}+\mathrm{X}_{234}+\mathrm{X}_{234} \mathrm{Y}$.

\section{References}

Epp, S. S. (2010). Discrete mathematics with applications. Cengage learning.

Leung, K. T., Suen, S. N., \& Mok, I. A. (1992). Polynomials and Equations: A Chinese Merchant Elite in Colonial Hong Kong (with a new preface) (Vol. 1). Hong Kong University Press.

Read, R. C. (1968). An introduction to chromatic polynomials. Journal of Combinatorial Theory, 4(1), 52-71. https://doi.org/10.1016/S0021-9800(68)80087-0

Satu, E. S. (2007). Graph clustering. Computer Science Review, 1(1), $27-64$. https://doi.org/10.1016/j.cosrev.2007.05.001

Sopena, E. (1993). On the chromatic number of oriented partial k-trees. Internal Report, University Bordeaux I, submitted.

Tutte, W. T. (1954). A contribution to the theory of chromatic polynomials. Canadian Journal of Mathematics, 6, 80-91. https://doi.org/10.4153/CJM-1954-010-9 


\section{Copyrights}

Copyright for this article is retained by the author(s), with first publication rights granted to the journal.

This is an open-access article distributed under the terms and conditions of the Creative Commons Attribution license (http://creativecommons.org/licenses/by/4.0/). 